\title{
Detection of Coherent Terahertz Radiation from a High-Temperature Superconductor Josephson Junction by a Semiconductor Quantum-Dot Detector
}

\author{
R. Shaikhaidarov, V. N. Antonov, and A. Casey \\ Physics Department, Royal Holloway University of London, Egham, Surrey TW20 OEX, United Kingdom \\ A. Kalaboukhov and S. Kubatkin \\ MC2, Chalmers University of Technology, S-41296 Göteborg, Sweden \\ Y. Harada and K. Onomitsu \\ NTT Basic Research Laboratories, Wakamia, Atsugi-shi, Kanagawa-ken 153-8902, Japan
}

A. Tzalenchuk

National Physical Laboratory, Hampton Road, Teddington, Middlesex TW11 OLW, United Kingdom and Physics Department, Royal Holloway University of London, Egham, Surrey TW20 OEX, United Kingdom

\author{
A. Sobolev \\ Kotel'nikov Institute of Radio Engineering and Electronics RAS, \\ Mokhovaya st. 11/7, Moscow 125009, Russia
}

(Received 23 August 2015; revised manuscript received 17 November 2015; published 22 February 2016)

\begin{abstract}
We examine the application of Josephson radiation emitters to spectral calibration of single-photonresolving detectors. Josephson junctions are patterned in a YBCO film on a bicrystal sapphire substrate and are voltage controlled to generate radiation in the frequency range of $0.05-1 \mathrm{THz}$. The detector used in this work consists of a gate-defined quantum-dot photon-to-charge transducer coupled to a single-electron transistor. Both the emitter and the detector are equipped with a matching on-chip wide-band antenna. The combination of a tuneable emitter and detector allows us to determine the efficacy of the YBCO emitter and also to analyze the elementary processes involved in the detection.
\end{abstract}

DOI: 10.1103/PhysRevApplied.5.024010

\section{INTRODUCTION}

Cryogenic detectors of terahertz radiation with singlephoton sensitivity have been demonstrated in recent years [1-5]. Spectral calibrations of these detectors have been carried out using external terahertz sources, narrow-bandfiltered thermal sources, or sources based on cyclotron emission from 2DEGs in strong magnetic field. None of these solutions are suitable when recurring calibrations in a wide range of frequencies are required to establish how the spectral characteristics of the detector have changed with time, but the payload is constrained in certain situations such as on a satellite. Here we examine a calibration solution based on a Josephson junction.

Voltage applied across a Josephson junction (JJ) causes oscillations of the dissipative current and, correspondingly, the generation of electromagnetic radiation, with a frequency $f=2 \mathrm{eV}_{\mathrm{JJ}} / h$. The upper limit of the frequency is determined by the value of the superconducting gap. In $S-I-S$ junctions, the gap is related to the critical current and the normal resistance of the JJ, $I_{C}$, and $R_{N}$ via the Ambegaokar-Baratoff relation, $I_{C} R_{N}=$ $(\pi / 2 e) \Delta_{S} \tanh \left(\Delta_{S} / 2 k_{B} T\right)$, which for $T \rightarrow 0$ becomes
$I_{C} R_{N}=(\pi / 2 e) \Delta_{S}$. In high-temperature superconductors (HTSs), $\Delta_{S}$ can be as large as $15 \mathrm{meV}$, which corresponds to $f \sim 7 \mathrm{THz}$. However, when the junction is shunted intrinsically, which is the case for HTS JJs, or extrinsically (by an external resistor), the radiation is suppressed below the gap value and is determined by $I_{C} R_{N}<\Delta_{S} / e$. Radiation emitted by HTS JJs has been the subject of intense research in recent years, motivated by the need for a spectral source of terahertz radiation. Individual JJs coupled to planar antennas are the simplest realization of such terahertz sources, and they can deliver a narrow radiation line [6] with continuous tuning of frequency over a wide range. The overall power dissipated by the individual HTS JJ is $I_{C} V_{\mathrm{JJ}}$, with the maximum $V_{\mathrm{JJ}}$ limited by $I_{C} R_{N}$. Part of this power is dissipated as Joule heating, and only a fraction, typically a few picowatts, is converted into coherent radiation $[7,8]$. In a planar HTS JJ, most of the coherent radiation remains inside the substrate, and only a small part is radiated into the environment because of mismatch of the dielectric constants between the substrate material and air or lens, and low coupling efficiency of the radiation of an individual $\mathrm{JJ}$ to the planar antenna. It is a formidable task to develop an accurate 
model of such a source of coherent radiation. An experimental assessment is also difficult because the coherent radiation is weak and obscured by the strong blackbody radiation of the heated JJ. One needs a spectrally sensitive detector which would be blind to the blackbody radiation in order to succeed. The purpose of this paper is to show that the weak radiation of individual JJs can be detected, and it can be useful in the spectral calibration of the cryogenic detectors.

It was recognized very soon after the discovery of the Josephson effect in 1962, that Josephson junctions can be used as a source of controllable coherent electromagnetic radiation at cryogenic temperatures. The first direct measurements of the emitted radiation were made by Yanson et al. [9] in 1965. The radiation power $W \sim 10^{-14} \mathrm{~W}$ was estimated in these early measurements on low- $T_{c}$ superconductors. This low power was attributed to a mismatch between the junction impedance and the impedance of vacuum. There have been only a few attempts to measure the coherent radiation emitted by a HTS JJ. A hot electron bolometer and a separate HTS JJ have been used as detectors [10-12]. In these experiments, the power of coherent radiation incident on a $0.5 \times 0.5 \mathrm{~mm}^{2}$ area of a bolometer was estimated to be $P_{\text {osc }} \sim 10^{-13} \mathrm{~W}$, when the source and sensor were placed back to back [10]. This result, however, has a high level of uncertainty because of an inherent problem with Joule heating affecting detectors and variability related to the samples. Further study of individual planar HTS JJs has stalled, as attention has recently focused on the properties of stacks of patterned intrinsic Josephson junctions in $\mathrm{Bi}_{2} \mathrm{Sr}_{2} \mathrm{CaCu}_{2} \mathrm{O}_{8}$. Emission of coherent radiation of up to $30 \mu \mathrm{W}$ with a full width at half maximum (FWHM) as small as $50 \mathrm{MHz}$ was reported [13-15]. The FWHM and central frequency in these devices are determined by the spatial resonance in the coupled JJs of the patterned stack. While demonstrating high power and a narrow line of emitted radiation, these devices have one substantial drawback: their limited frequency tuning, less than $10 \%$, which is achieved by varying the operation temperature.

In this work, we return to the operation of an individual JJ. While inferior to stacked Josephson junctions in terms of the output power, they have an advantage in their ability to continuously tune the output frequency over a wide range. The HTS JJ in our device is coupled to a planar metallic logarithmic periodic antenna with a designed bandwidth of $0.2-2 \mathrm{THz}$. When a bias voltage is applied across the $\mathrm{JJ}$, it generates radiation in a frequency range up to $1 \mathrm{THz}$. The JJ radiation is probed by a quantum-dot detector which has a single-photon sensitivity enabling spectroscopy of extremely low-power coherent radiation. We observe two resonances in the photoresponse, at approximately 0.3 and $0.8 \mathrm{THz}$, which can be attributed to an excitation of the resonance plasma oscillations in the detector. The resonances are relatively wide, approximately
$30 \%$, which is usual for the plasma excitations in a semiconductor material. It exceeds the FWHM of the coherent radiation of a HTS JJ, which typically is $1 \mathrm{GHz}$ for individual JJs [11]. We find that only a tiny part, $4 \times 10^{-10}$, of the power dissipated in the HTS JJ is emitted as coherent radiation into vacuum. No particular resonances related to the planar antennas or the shape of the HTS JJ are seen.

\section{EXPERIMENTAL RESULTS AND DISCUSSION}

The experiments are carried out using two different measurement setups. The HTS JJs and quantum-dot (QD) detectors in both experiments are identical. In the first setup, the HTS JJ and the QD detector are at $0.02 \mathrm{~K}$ of the mixing chamber of a dilution refrigerator. The HTS JJ is fixed on a semispherical $\mathrm{Si}$ lens coupled to a $5-\mathrm{cm}$ light pipe, a stainless-steel tube of $3 \mathrm{~mm}$ diameter, extended to the QD detector; see the inset in Fig. 1. The attenuation of the optical system is estimated to be $22 \mathrm{~dB}$ if we assume the quantum efficiency of the detector to be about 0.003 [16]. In our estimation, we take the impedance of the QD and the antenna to be 300 and $10 \Omega$ at $0.3 \mathrm{THz}$, respectively. In the second experimental setup, a similar HTS JJ is fixed at the 4-K stage of a cryogen-free refrigerator in close proximity to the QD detector. Radiation emitted by the HTS JJ is delivered to the detector via the light pipe described above. A black polyethylene filter is used to filter out the infrared radiation. The QD detector is fixed in a metal enclosure at the $0.5-\mathrm{K}$ stage.

\section{A. HTS Josephson junction emitter}

A grain-boundary junction integrated with a logarithmic periodic antenna is patterned by photolithography into a 200-nm-thick YBCO film. The YBCO is grown by pulsedlaser deposition on a sapphire bicrystal substrate with the misorientation angle of $24^{\circ}$ [17]. The junction has a width of $2 \mu \mathrm{m}$, and the antenna has an outer diameter of $0.5 \mathrm{~mm}$;

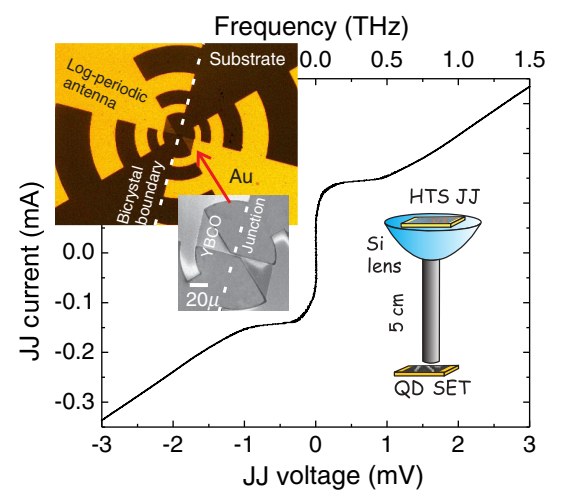

FIG. 1. $I-V$ curve of a HTS JJ taken at $0.02 \mathrm{~K}$. Top axis is the Josephson frequency corresponding to the voltage across the JJ. Insets: Optical image of a $2-\mu$ m-wide HTS JJ defined at the bicrystal boundary and the optical scheme of the experiment. 
see the inset in Fig. 1. A typical current voltage $(I-V)$ characteristic of a HTS JJ is shown in Fig. 1. The shape of the $I-V$ curve indicates that the $\mathrm{JJ}$ is overdamped. There are no obvious resonances on the $I-V$ curve related to the coupling of the $\mathrm{JJ}$ to the metallic planar antennas. The $I_{C} R_{N}$ product of a typical $\mathrm{JJ}$ is $3.8 \mathrm{mV}$, and, therefore, a coherent radiation of up to $2 \mathrm{THz}$ can be generated. Only a small part of the dissipated power is, however, converted to coherent radiation at the Josephson frequency, while the largest part is dissipated via phonons and blackbody radiation. The latter becomes especially critical when the voltage across the JJ exceeds approximately $1 \mathrm{mV}$, as the HTS JJ microbridge is then strongly overheated. The effective temperature of the microbridge $T_{m}$ can be estimated using a simplified model $T_{m}=$ $\sqrt{T_{b}^{2}+3\left(e V_{\mathrm{JJ}} / 2 \pi k_{B}\right)^{2}}$, where $T_{b}$ is the bath temperature [18]. For a $V_{\mathrm{JJ}}$ bias of $1 \mathrm{mV}$, the effective temperature $T_{m}$ can be as high as $3 \mathrm{~K}$, while the bath temperature $T_{b}$ is only $0.26 \mathrm{~K}$ [9]. Overheating results in an increase in the blackbody radiation emitted by the HTS JJ. Because $T_{m}$ grows linearly with $V_{\mathrm{JJ}}$, the incoherent blackbody radiation dominates over the coherent radiation at high bias voltages. In order to separate the two components of the radiation, one needs a spectrally sensitive detector, which will detect only the coherent part of radiation. In Ref. [12], a separate individually tuned HTS JJ detector was used for such an experiment. However, the analysis was inconclusive because of the presence of a bolometric response of the detector to blackbody radiation. We utilize the QD detector, which is robust against blackbody radiation when operating in the regime of excitation of plasma resonance in the QD.

\section{B. Quantum-dot detector}

The detector is a semiconductor QD coupled to an aluminum single-electron transistor (SET). The QD is positioned at the focal point of a log-periodic antenna. The QD of a diameter $D \sim 1.4 \mu \mathrm{m}$ is defined by a mesa pattern and a biased metal gate; see the inset in Fig. 2. The 2DEG has the electron concentration of $n_{s} \sim 1.45 \times$ $10^{11} \mathrm{~cm}^{-2}$ and mobility $\mu \sim 1.2 \times 10^{5} \mathrm{~cm}^{2} /$ Vs at $T=4 \mathrm{~K}$. The QD has a few thousand electrons, and it can be considered a semiclassical object in which plasma oscillations can be excited. These oscillations provide an efficient way of coupling terahertz radiation to the electron system in a semiconductor. From the mobility of the electron gas, we can estimate the damping factor of the plasma oscillations $1 /(2 \pi) f \tau_{e}$, which is smaller than 0.15 at $f=0.25 \mathrm{THz}$, where $\tau_{e}$ is an electron-scattering time. Therefore, we can expect excitation of the plasma mode in the QD upon absorption of terahertz photons. The fundamental mode of plasma oscillations in the dot is determined by its diameter $D$ and the carrier concentration $n_{s}$, $f^{2}=e^{2} n_{s} / \pi^{2} \varepsilon_{\text {eff }} m^{*} D$, where $\varepsilon_{\text {eff }} \sim 7$ is the effective

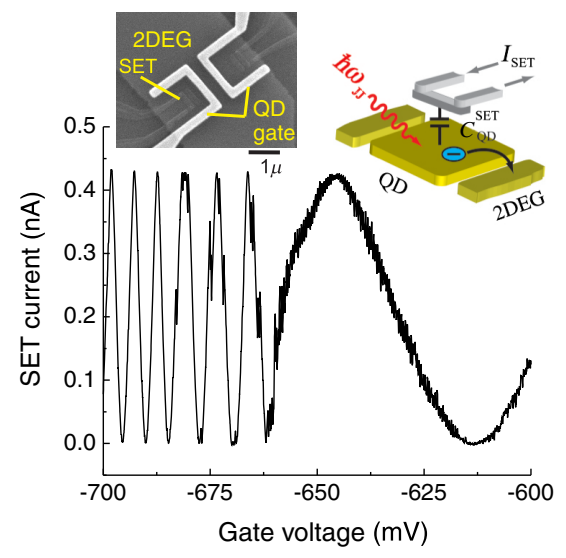

FIG. 2. CBO of the aluminum SET as a function of voltage on the QD gate. The CBO period changes at $V_{G} \sim-0.654 \mathrm{~V}$ when the QD is formed in the 2DEG. Inset: A SEM picture and operation scheme of the detector. The QD is formed in the 2DEG by the negatively biased QD gate and the edge of the mesa strip. The QD is capacitively coupled to the aluminum SET fabricated on top of the mesa strip. The SET current is sensitive to the excitation of electrons out of the QD upon absorption of terahertz photons.

dielectric constant at the vacuum-GaAs interface. For our device, the formula gives the resonance frequency of $f \sim 0.23 \mathrm{THz}$. Plasma resonances in a QD of a slightly different size and design have been seen before [19]. In a QD of $D \sim 0.5 \mu \mathrm{m}$ and $n_{s}=0.9 \times 10^{11} \mathrm{~cm}^{-2}$, the resonance is at $f \sim 0.5 \mathrm{THz}$ with the FWHM-to- $f$ ratio of $30 \%$. However estimation of the resonance frequency from the formula gives a smaller value, about $70 \%$ of that measured, which can be attributed to an uncertainty in the determination of the QD size. The FWHM decreases substantially, down to $5 \%$, when a moderate magnetic field of $1 \mathrm{~T}$ is applied. The magnetoplasma resonance is then excited [20]. The QD is not sensitive to radiation with frequencies away from the resonance. The Drude absorption by the QD, the other photon-absorption mechanism, is much weaker because of a large mismatch of the radiation wavelength and the size of the dipole related to individual electrons. Consequently, the blackbody radiation of the overheated JJ, which will peak at $50 \mathrm{GHz}$, has a small effect on the operation of the QD detector. Indeed we observe the decrease of the photoresponse at high bias $V_{\mathrm{JJ}}$, where $f=$ $2 e V_{\mathrm{JJ}} / h$ exceeds the plasma resonance in the $\mathrm{QD}$, while the blackbody radiation power is on the rise.

The large FWHM of the plasma resonance implies that the lifetime of the excitation in the QD at zero magnetic field is short, only a few picoseconds. The plasma oscillations decay in a way that either charge polarization is induced in the QD or even charge is excited out of the QD. Polarization of the charge density in the 2DEG is used in plasmonic detectors [4,21-23]. In our experiment, we consider the second mechanism-charge excitation of the QD. The detector is designed in such way that charge 
excitations of the QD are probed by a sensitive electrometer (SET). Details of the fabrication and the operation of the SET can be found elsewhere $[24,25]$. Coulomb blockade oscillations (CBOs) of the SET current are shown in Fig. 2. The period of the oscillations changes from 62.7 to $5.28 \mathrm{mV}$ when the $\mathrm{QD}$ is formed at $V_{G} \sim-0.654 \mathrm{~V}$. The pattern of CBO is shifted by $\delta V_{G}=$ $C_{\mathrm{SET}}^{\mathrm{QD}} /\left(C_{\Sigma}^{\mathrm{QD}}+C_{\mathrm{SET}}^{\mathrm{QD}}\right) \delta V_{G}^{\text {period }}$ every time an electron leaves the QD because of the capacitive coupling $C_{\mathrm{SET}}^{\mathrm{QD}}$ between the SET and the QD, where $C_{\Sigma}^{\mathrm{QD}}$ is the total capacitance of $\mathrm{QD}$, and $\delta V_{G}^{\text {period }}$ is the CBO period. For the present device, the shift is approximately $20 \%$ of $\delta V_{G}^{\text {period }}$ [24]. When the gate voltage $V_{G}$ is fixed, then the electron excitations are seen as steplike changes of the SET current; see Fig. 5(a). One can find the rate and the lifetime of the QD excitations by counting the number of SET current spikes and measuring the time spent in the excited state. The spikes are more frequent close to the gate voltage where the QD is just formed, $V_{G} \sim-0.654 \mathrm{~V}$, due to excitation of thermal electrons. These excitations are suppressed at more negative $V_{G}$ when a high-enough potential barrier is imposed by the gate voltage; see Fig. 3. Close to the pinch-off voltage, the count rate can be modeled by an exponential decay of the form, $P=P_{0} \exp \left[-a\left(V_{G}-V_{G 0}\right) / k_{B} T\right]$, with $V_{G 0}=-0.654 \mathrm{~V}$ being the threshold voltage where the QD is formed. The change in the electrochemical potential of electrons and the applied gate voltage are related to each other by $\delta \mu=a \delta V_{G}$, where $a=13.8 \mathrm{meV} / \mathrm{V}$. We use the value of $a$ later in the analysis. It is assumed that the tunneling of electrons under the barrier is negligible because of the large width of the gate electrode. Then the charge excitations from the QD should take place only when the electron energy exceeds the potential barrier, for example, in the case of absorption of a photon by the QD. Therefore, the most sensitive operation of the detector is

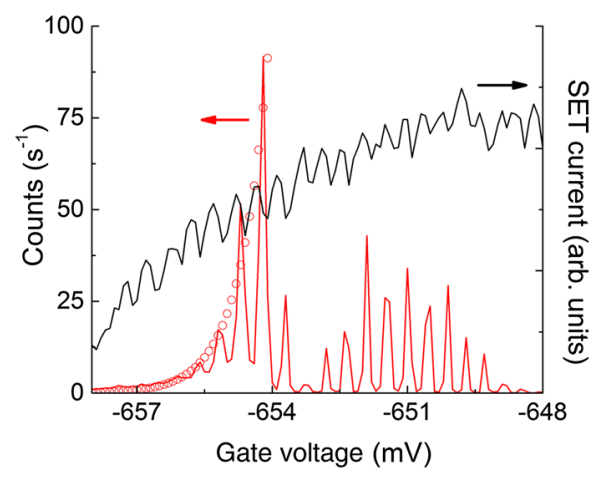

FIG. 3. Count rate of charge excitation of the QD due to thermal photons at $T=0.02 \mathrm{~K}$. Oscillations of the count rate are correlated with the CBO of the QD seen in the SET current. Counts are suppressed below $V_{G} \sim-0.657 \mathrm{~V}$ when the QD is completely decoupled from the surrounding 2DEG. Open circles are the exponential fit of the suppression expected below $-0.654 \mathrm{~V}$. The SET current and the count rate coherently oscillate, as seen in Fig. 3. The effect is a consequence of the Coulomb blockade of the electron excitations from the QD itself.

\section{Detection of HTS JJ radiation}

We measure the count rate with a fixed $V_{G}$, while changing the voltage across the HTS JJ. The results are shown in Fig. 4. There are two clear peaks with FWHM of $30 \%$ and central frequencies close to $f \sim 0.3 \mathrm{THz}$ and $f \sim 0.8 \mathrm{THz}$. The counting rate drops rapidly at frequencies above $0.9 \mathrm{THz}$ and below $0.1 \mathrm{THz}$. It is important to note that the count rate drops at large bias voltages $V_{\mathrm{JJ}}$, where incoherent blackbody radiation of the overheated HTS JJ should contribute to the photoresponse. This contribution was seen in the experiment of Ref. [10], where a bolometer was used. The photoresponse increased there as the square of the bias voltage, $V_{\mathrm{JJ}}^{2}$, because the bolometer absorbs blackbody radiation emitted by the overheated JJ. We do not observe this effect, which clearly indicates that our detector does not respond to blackbody radiation but rather detects coherent radiation at frequency $f$. The photoresponse is seen, however, only in a narrow range of gate voltages $V_{G}$, from -0.654 to $-0.657 \mathrm{~V}$. By using these voltages, we can estimate the change of the effective potential in the vicinity of the $2 \mathrm{DEG}$ and, consequently, the increase of the barrier height confining electrons on the $\mathrm{QD}, \delta U=a \delta V_{G} \sim 40 \mu \mathrm{eV}$. This change is much smaller than the energy of the terahertz photons, $1 \mathrm{meV}$ for $f=0.3 \mathrm{THz}$. One expects that photoexcitations should extend deep into negative $V_{G}$, which is not seen in the experiment. The effect can hardly be ascribed to a substantial change of the coupling of radiation to the QD. Probably, it is a consequence of the suppression of the relaxation mechanism at low temperatures. If the tunneling of electrons from the 2DEG electrodes into the QD is suppressed, then the QD can be multiply excited by absorbing consecutive photons. For a multiexcited QD, the energy of a terahertz photon may not be enough to

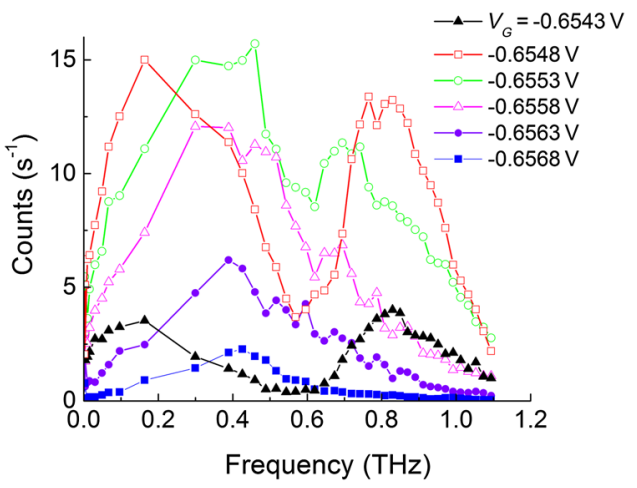

FIG. 4. Count rate of the QD detector under radiation emitted by a HTS JJ at different $V_{G}$. There are two peaks in the count rate, one close to $0.3 \mathrm{THz}$ and the other around $0.8 \mathrm{THz}$. 
overcome the potential barrier forming the QD. We can estimate the order of excitation needed for such a situation by invoking the charging energy of the $\mathrm{QD}$, approximately $80 \mu \mathrm{eV}$ [24]. Excitation of each electron out of the QD decreases the potential of the electrons in the QD by $80 \mu \mathrm{eV}$ so that the excitation of a dozen electrons should completely block further excitation. The energy of the absorbed photons can then be released by nonradiative phonon emission.

The frequency of the first resonance varies with the $V_{G}$ from $0.18 \mathrm{THz}$ at $V_{G}=-0.6543 \mathrm{~V}$ to $0.4 \mathrm{THz}$ at $V_{G}=-0.6568 \mathrm{~V}$. We estimate that the plasma resonance frequency in our device should be $0.23 \mathrm{THz}$. Since the resonance frequency varies as the square root of the size, the diameter of the QD should change by a factor of 4 in order to account for the observed frequency change. Such a dramatic effect can be explained only by the assumption that at small negative $V_{G}$ the QD is not well defined, and the potential barriers are shallow, such that plasma waves of long wavelength can be excited. As $V_{G}$ becomes more negative, the potential barrier becomes steeper, and the resonance appears to be better defined by the geometrical dimensions. The opposite trend is demonstrated by the resonance peak at high frequency. It moves towards the lower frequencies, from $0.85 \mathrm{THz}$ at $V_{G}=-0.6543 \mathrm{~V}$ to approximately $0.7 \mathrm{THz}$ at $V_{G}=-0.6558 \mathrm{~V}$, and then disappears; see Fig. 4.

One can get further insight into the resonances from a more accurate analysis of the switching time traces, see Fig. 5, which allows deconvolution of various (de-) excitation processes of the QD. When the voltage across the HTS JJ corresponds, for example, to $0.8 \mathrm{THz}$, one can identify five states of the QD from Fig. 5(a): ground states $A$ and $-A$ (rigorous identification of the true ground state is impossible because these two states appear nearly as frequently in the time traces), first excited states $B$ and $-B$ corresponding to the excitation of one electron out or onto the $\mathrm{QD}$, and the second excited state $-C$ corresponding to the excitation onto the QD. The neighboring states differ by one electron on the QD. We repeat this procedure
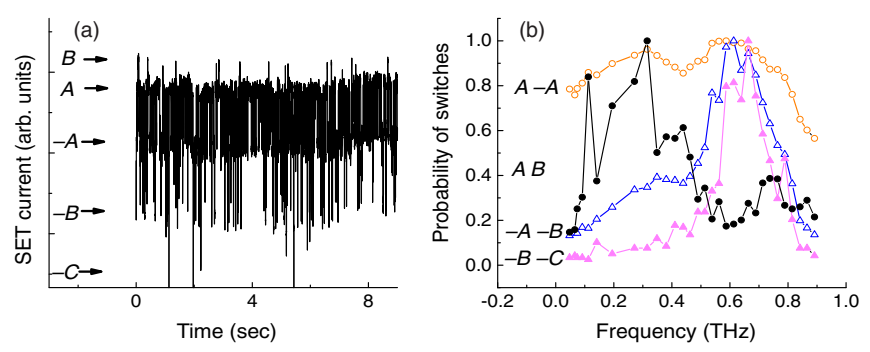

FIG. 5. (a) Time traces of SET current at $0.8 \mathrm{THz}$. There are few levels corresponding to different QD states: ground $A$ and $-A$, excited $B$ and $-B$, double excited $-C$. (b) Switches between different states normalized to maximum counts: $A B$ switches have a maximum at $0.3 \mathrm{THz},-A-B$ and $-B-C$ have a maximum at $0.7 \mathrm{THz}$, and $A-A$ has both resonances. for various $V_{\mathrm{JJ}}$ 's. The count rates in Fig. 4 are a sum of all excitations: $-A A,-A-B,-B-C$, and $A B$, while in Fig. 5(b) we count each type of excitation separately. The $A-A$ excitation has two peaks at 0.3 and $0.7 \mathrm{THz}$ on a background of a high rate of dark switches. The $-A-B$ and $-B-C$ excitations have a dominant resonance peak at $0.7 \mathrm{THz}$, while the $A B$ excitations have a peak at $0.3 \mathrm{THz}$. The sign of the SET current switches indicates that electrons are predominantly excited out of the QD at the $0.3-\mathrm{THz}$ resonance, and they are excited into the QD at $0.8 \mathrm{THz}$. There are two possible origins of the $0.8-\mathrm{THz}$ peak: a high-order plasma mode in the QD or the resonance plasma waves developed outside of the QD. The latter can be a plasma wave developed in the 2DEG between two QD gates; see the left inset in Fig. 2. The distance between the gates is $0.2 \mu \mathrm{m}$, which will give $f \sim 0.6 \mathrm{THz}$ for the central frequency. The fact that electrons are excited to the QD at this resonance supports the explanation.

From our experiment, we can estimate the ratio between coherent radiation and the total dissipated power of the HTS JJ. A typical increase of the counting rate at the resonance compared to the background is $10 \mathrm{~s}^{-1}$. The power of the coherent radiation detected by the QD SET detector is $2 \times 10^{-21} \mathrm{~J}$ for $f \sim 0.3 \mathrm{THz}$. If the attenuation of the optical system is $22 \mathrm{~dB}$, then the power emitted by the HTS JJ to the Si lens is $30 \mathrm{aW}$. The HTS JJ dissipates a total power of $80 \mathrm{nW}$; thus, only the $4 \times 10^{-10}$ part is emitted as coherent radiation to the Si lens. A few factors can be considered in order to account for such a small value. A large part of the radiation is emitted along the surface of the substrate and does not enter the Si lens at all. Up to a quarter of the radiation stays in the JJ itself, and a few percent is emitted into vacuum opposite the Si lens. Also, we cannot accurately account for the polarization of the emitted radiation with respect to the detector when we estimate the quantum efficiency.

\section{SUMMARY}

We study radiation emitted by HTS JJ using a QD detector. The QD detector allows us to probe the coherent part of the radiation. Two resonances are seen in the photoresponse when we sweep voltage across the JJ, at approximately 0.3 and $0.8 \mathrm{THz}$. We argue that the lower resonance is related to excitation of plasma waves in the QD of the detector, while the origin of the second resonance is not exactly clear. Coherent radiation is only a $4 \times 10^{-10}$ part of the total power dissipated by the JJ. We believe that the small conversion factor is partially related to the weak coupling of the HTS JJ to the planar antennas, a difference in the dielectric constant of the HTS JJ substrate and vacuum or lens material, and a possible polarization mismatch. Although the individual HTS JJ coupled to a radiating antenna has an extremely low conversion efficiency of power to the coherent radiation, the emitters based on these devices can be useful for spectral calibration 
of cryogenic single-photon detectors, especially where recurring system self-calibration is required, and no other means would do, such as on a satellite. In addition, they may also be useful as generators of coherent photons in applications to cryogenic quantum simulators and computers. In applications where higher power is required alongside the wide-range tunability of individual JJs, phase-locked parallel-biased series arrays of HTS JJs may be employed [26,27] (also, see Ref. [28] for a review of JJ array radiation sources).

\section{ACKNOWLEDGMENT}

The work is supported by EPSRC Grant No. EP/ K016822/1.

[1] S. Komiyama, O. Astafiev, V. Antonov, T. Kutsuwa, and H. Hirai, A single-photon detector in the far-infrared range, Nature (London) 403, 405 (2000).

[2] H. Hashiba, V. Antonov, L. Kulik, A. Ya. Tzalenchuk, P. Kleinschmid, S. Giblin, and S. Komiyama, Isolated quantum dot in application to terahertz photon counting, Phys. Rev. B 73, 081310(R) (2006).

[3] P. Kleinschmidt, S. Giblin, A. Ya. Tzalenchuk, H. Hashiba, V. Antonov, and S. Komiyama, Sensitive detector for a passive terahertz imager, J. Appl. Phys. 99, 114504 (2006).

[4] S. Pelling, R. Davis, L. Kulik, A. Tzalenchuk, S. Kubatkin, T. Ueda, S. Komiyama, and V. N. Antonov, Point contact readout for a quantum dot terahertz sensor, Appl. Phys. Lett. 93, 073501 (2008).

[5] H. Hashiba, V. Antonov, L. Kulik, A. Tzalenchuk, and S. Komiyama, Sensing individual terahertz photons, Nanotechnology 21, 165203 (2010).

[6] Y. Y. Divin, J. Mygind, N. F. Pedersen, and P. Chaudhari, Linewidth of Josephson oscillations in $\mathrm{YBa}_{2} \mathrm{Cu}_{3} \mathrm{O}_{7-x}$ grainboundary junctions, IEEE Trans. Appl. Supercond. 3, 2337 (1993).

[7] J. Du, J. C. Macfarlane, T. Zhang, Y. Cai, and Y. J. Guo, Self-pumped HTS Josephson heterodyne tunable mixer, Supercond. Sci. Technol. 25, 025019 (2012).

[8] J. Du and J. C. Macfarlane, Frequency down-conversion in self-pumped HTS R-SQUID mixer, Electron. Lett. 47, 772 (2011).

[9] I. K. Yanson, V. M. Svistunov, and I. M. Dmitrenko, Experimental observation of tunnel effect for cooper pairs with emission of photons, JETP 21, 650 (1965).

[10] E. Stepantsov, M. Tarasov, A. Kalabukhov, L. Kuzmin, and T. Claeson, $\mathrm{THz}$ Josephson properties of grain boundary $\mathrm{YBaCuO}$ junctions on symmetric, tilted bicrystal sapphire substrates, J. Appl. Phys. 96, 3357 (2004).

[11] I. E. Batov, X. Y. Jin, S. V. Shitov, Y. Koval, P. Mller, and A. V. Ustinov, Detection of $0.5 \mathrm{THz}$ radiation from intrinsic $\mathrm{Bi}_{2} \mathrm{Sr}_{2} \mathrm{CaCu}_{2} \mathrm{O}_{8}$ Josephson junctions, Appl. Phys. Lett. 88, 262504 (2006).

[12] M. Tarasov, E. Stepantsov, T. Lindstrom, A. Lohmus, L. Kuzmin, and Z. Ivanov, Submillimeter-wave quasioptical integrated tester based on bicrystal Josephson junctions, Physica (Amsterdam) 372C-376C, 347 (2002).

[13] L. Ozyuzer, A. E. Koshelev, C. Kurter, N. Gopalsami, Q. Li, M. Tachiki, K. Kadowaki, T. Yamamoto, H. Minami, H. Yamaguchi, T. Tachiki, K. E. Gray, W.-K. Kwok, and U. Welp, Emission of coherent $\mathrm{THz}$ radiation from superconductors, Science 318, 1291 (2007).

[14] I. Kakeya, Y. Omukai, T. Yamamoto, K. Kadowaki, and M. Suzuki, Effect of thermal inhomogeneity for terahertz radiation from intrinsic Josephson junction stacks of $\mathrm{Bi}_{2} \mathrm{Sr}_{2} \mathrm{CaCu}_{2} \mathrm{O}_{8+\delta}$, Appl. Phys. Lett. 100, 242603 (2012).

[15] D. Y. An et al., Terahertz emission and detection both based on high- $T_{c}$ superconductors: Towards an integrated receiver, Appl. Phys. Lett. 102, 092601 (2013).

[16] O. Astafiev and S. Komiyama, in Electron Transport in Quantum Dots, edited by J. Bird (Kluwer, Dordrecht, 2003).

[17] E. Stepantsov, M. Tarasov, A. Kalabukhov, T. Lindstrom, Z. Ivanov, and T. Claeson, Submicron YBCO Josephson junctions on sapphire bicrystal substrates for microwave devices, Physica (Amsterdam) 372C-376C, 76 (2002).

[18] M. Tihkham, M. Octavio, and W. Scocpol, Heating effects in high-frequency metallic Josephson devices: Voltage limit, bolometric mixing, and noise, J. Appl. Phys. 48, 1311 (1977).

[19] O. Astafiev, S. Komiyama, T. Kutsuwa, V. Antonov, Y. Kawaguchi, and K. Hirakawa, Single-photon detector in the microwave range, Appl. Phys. Lett. 80, 4250 (2002).

[20] O. Astafiev, V. Antonov, T. Kutsuwa, and S. Komiyama, Far-infrared spectroscopy of single quantum dots in high magnetic fields, Phys. Rev. B 65, 085315 (2002).

[21] V. M. Muravev and I. V. Kukushkin, Plasmonic detector/ spectrometer of subterahertz radiation based on twodimensional electron system with embedded defect, Appl. Phys. Lett. 100, 082102 (2012).

[22] F. Teppe, W. Knapp, D. Veksler, M. S. Shur, A. P. Dmitriev, V. Yu. Kachorovskii, and S. Rumyantsev, Roomtemperature plasma waves resonant detection of subterahertz radiation by nanometer field-effect transistor, Appl. Phys. Lett. 87, 052107 (2005).

[23] J. W. Song, N. A. Kabir, Y. Kawano, K. Ishibashi, G. R. Aizin, L. Mourokh, J. L. Reno, A. G. Markelz, and J. P. Bird, Terahertz response of quantum point contacts, Appl. Phys. Lett. 92, 223115 (2008).

[24] S. Pelling, E. Otto, S. Spasov, S. Kubatkin, R. Shaikhaidarov, K. Ueda, S. Komiyama, and V. N Antonov, Electrostatic effects in coupled quantum dot-point contact-single electron transistor devices, J. Appl. Phys. 112, 014322 (2012).

[25] M. H. Devoret and R. J. Schoelkopf, Amplifying quantum signals with the single-electron transistor, Nature (London) 406, 1039 (2000).

[26] J. Edstam, G. Brorsson, E. A. Stepantsov, and H. K. Olsson, Integrated high- $T_{c}$ oscillator array and high resistance detector junction, IEEE Trans. Appl. Supercond. 5, 3276 (1995).

[27] J. Edstam, P. Larsson, E. A. Stepantsov, and H. K. Olsson, Phase locking of an array of five high- $T_{c}$ Josephson junction oscillators, Physica (Amsterdam) 235C-240C, 3385 (1994).

[28] M. Darula, T. Doderer, and S. Beuven, Millimetre and sub-mm wavelength radiation sources based on discrete Josephson junction arrays, Supercond. Sci. Technol. 12, R1 (1999). 